\title{
TENDENCIAS ACTUALES Y FUTURAS EN LA DIVISIÓN INTERNACIONAL DEL TRABAJO. ASPECTOS ECONÓMICOS, TECNOLÓGICOS Y POLÍTICOS*
}

\author{
GERARD K. BOON \\ El Colegio de México y \\ The Technology Scientific Foundation
}

\section{INTRODUCCIÓN}

LA DIVISIÓN INTERNACIONAL DEL TRABAJO, sobre todo entre las naciones del tercer y el primer mundos, es un tema importante en la economía política actual de los países en desarrollo y los desarrollados, y en consecuencia, en el diálogo Norte-Sur. No habrá gran diferencia de opiniones en cuanto al hecho de que este aspecto, la especialización de la producción internacional entre los países, está influido por numerosos factores, y como materia de estudio tiene, entre otros, importantes aspectos económicos, tecnológicos y políticos. En este resumen se mencionan y se analizan brevemente algunos elementos de estos aspectos.

La teoría clásica del desarrollo económico dedicó especial atención al comercio internacional sobre la base de los costos absolutos y en particular de los costos comparativos de producción (Ricardo). De la misma manera, el progreso tecnológico ha sido una materia a la que ya fueron atraídos los economistas clásicos. Estos dos temas se cuentan entre los tópicos económicos más antiguos, que han suscitado gran interés analítico desde el mismo principio del pensar económico.

No obstante, el fundamento teórico económico de un comercio internacional libre de obstáculos entre los países, que conduce a un grado óptimo de bienestar internacional, es puesto en duda por los políticos prácticos en la realidad actual, y de hecho puede surgir la interrogante sobre si este modelo podría ofrecer los resultados esperados. La teoría del comercio internacional, incluidos los principios de la división del trabajo entre los países, me parece reflejar en gran medida un orden mundial que era una realidad en el momento en que se formuló esta teoría, pero que ahora refleja mucho menos atinadamente la realidad política. Europa ha dejado de ser el centro del poder mundial al que casi todo el resto de las naciones esté subordinado. En mi opinión, en el caso de que un área del mundo domine a las otras, la división internacional del trabajo basada en la ventaja comparativa funciona perfectamente.

* Trabajo presentado a la sesión IV del Sexto Congreso Mundial de la Asociación Internacional de Economía, celebrada en la ciudad de México, agosto de 1980. Este documento es resumen de un trabajo más amplio. (Traducción del inglés de Beatriz Guiza). 
En la actualidad, todas las antiguas colonias europeas son estados independientes; además han surgido cuatro centros de poder: los Estados Unidos, la URSS y, en grado un tanto más modesto, Europa Occidental y Japón. De hecho, la realidad política internacional es sustancialmente diferente ahora, aunque todavía existen las dependencias básicas tecnológicas y económicas entre el tercer y el primer mundos, lo que entraña una dependencia política fundamental. Esta es precisamente la contradicción entre la independencia política de derecho del Tercer Mundo y la dependencia de hecho que, me parece, conduce a peligrosos sentimientos de frustración en el Tercer Mundo y, en consecuencia, hace que las relaciones internacionales económicas y políticas de esta área sean fundamentalmente inestables. Este hecho constituye un factor importante en la escena mundial de la política, lo que evidentemente deja también sentir su influencia en las relaciones llamadas Este-Oesie.

\section{ASPECTOS ECONÓMICOS*}

Si pudieran funcionar libremente las fuerzas del comercio internacional, el modelo básico de desarrollo del Tercer Mundo resultaría en un aprovechamiento de los recursos naturales agrícolas y minerales, y en exportaciones de estos sectores a cargo del "Sur", a cambio de productos industriales y tecnología del "Norte". Además las empresas trasnacionales penetrarían profundamente en el proceso del desarrollo del Tercer Mundo. Este modelo conduciría a una inaceptable dependencia del Sur en relación con el Norte: el hecho de calificar este tipo de relación como interdependiente es, en mi opinión, poco realista. La respuesta del Tercer Mundo para superar esta situación difiere de acuerdo con las diversas opciones abiertas para cada país o área.

Los Estados insulares y peninsulares que se encuentran en los contornos de Asia oriental han optado por integrarse a la economía mundial. Han abierto sus puertas a las empresas trasnacionales y a la tecnología compleja. De esta manera se convierten en una especie de apéndice del Primer Mundo, teniendo como resultado una alta tasa de crecimiento y, de acuerdo con ciertos criterios, un gran éxito en su desarrollo. Sin embargo, estos Estados tienen pocas alternativas y han tomado, sin duda, una decisión inteligente dadas sus limitaciones.

En América Latina prevalece otro modelo. Se ha buscado la vía de un desarrollo de tipo capitalista mediante la sustitución de importaciones de bienes de consumo, intermedios y de capital. La integración, mediante exportaciones, a la economía mundial ha sido permitida apenas en forma selectiva y parcial, y menos aún en lo que se refiere a importaciones. El área ha tenido un éxito relativo al seguir esta trayectoria, sin embargo, ha dado como resultado un desarrollo desequilibrado, y ha sido menos afortunada en cuanto a la integración de las economías rurales y semirurales, to que ha conducido a un desarrollo global insatisfactorio e inaceptable, con peligrosas inestabilidades intrínsecas.

Los grandes y densamente poblados países de Asia, China e India, han sido más afortunados al buscar un camino de desarrollo pluralista y, por lo tanto, han

* Véanse las referencias 1 a 5 . 
integrado sus economías en forma más equilibrada mediante eslabonamientos selectivos con la economía mundial.

En Africa existen todavía importantes vínculos con los antiguos y los nuevos agentes coloniales, con la dependencia que esto supone.

De estos cuatro amplios modelos de desarrollo, el segundo y el tercero, para América Latina e India y China, difieren considerablemente de lo que hubiera resultado de las libres fuerzas del mercado en el comercio internacional. Las economías industrializadas de mercado tampoco siguen las reglas del libre juego del libre comercio. El Mercado Común Europeo (CEE) constituye un buen ejemplo de lo que es un área de comercio manipulado. Evidentemente, la comunidad de países socialistas (CAME) se adhiere aún menos a las leyes del libre comercio internacional, aunque en lo que se refiere a productos para los que existe un mercado mundial, ellos siguen las reglas del juego y las estudian cuidadosamente con el fin de utilizarlas para su mayor provecho. Además, la política comercial práctica que aplican los Estados Unidos se desvia sustancialmente del modelo teórico capitalista.

La teoría clásica del comercio internacional y la división internacional del trabajo subrayan la diferencia de costos de mano de obra y de capital como principales motores del comercio. En la práctica, las diferencias en los costos del trabajo son verdaderamente muy importantes, ya que en términos reales, varían mucho más a nivel internacional que los costos de capital.

Sin embargo, sobre la base de mi reciente trabajo empírico en relación con la selección y transferencia de tecnología, y sus consecuencias en el empleo en las industrias del calzado, las fibras de poliéster, los hilados, los tejidos, el vestido y los productos metálicos, parece que el criterio del costo diferencial de la mano de obra y la supuesta división intemacional del trabajo de los productos conforme a la densidad relativamente alta de mano de obra en los paises en desarrollo y de capital en las naciones desarrolladas, necesita ciertas modificaciones.*

Las pruebas reunídas en relación con los productos mencionados demuestran que los de mayor intensidad de mano de obra constituyen la especialidad de la producción del mundo industrializado, en tanto que los artículos de menor intensidad de mano de obra corresponden al mundo en desarrollo. Consecuentemente, se presenta una paradoja en relación con el uso intensivo de mano de obra. No se llegó a esta conclusión analizando los modelos de comercio o producción, sino que fue a partir del análisis de la capacidad tecnológica de la maquinaria y los equipos preferidos en ambas áreas y de las características físicas complementarias de la producción. Resultó que los productos con mayores características comunes que son demandados y por lo tanto producidos en grandes cantidades por la industria de alta densidad, relativa, de capital por trabajador y con llamada maquinaria automatizada, se han desplazado en su mayor parte a las regiones que tienen costo de mano de obra relativamente bajo. Parece que el mundo desarrollado se ha concentrado en una especialidad de producción caracterizada por propiedades físicas más complejas y de elevada calidad que, conforme a la mercancía, puede ir de acuerdo a la moda y producirse en una gran-variedad de tipos

* Véanse las referencias 4 a 6. 
de calidades y colores. Estos últimos productos, que se fabrican en los lotes más pequeños, requieren de trabajo altamente calificado y se producen con densidad relativamente alta de mano de obra. Son precisamente la destreza y la habilidad que se requieren en la manufactura de estos artículos lo que falta por lo general en el Tercer Mundo, razón por la cual éste se especializa en la producción de bienes con menor densidad de mano de obra y habilidad manual, y mayor producción en serie.

Por lo tanto, la división internacional del trabajo, de acuerdo con estas evidencias, está mucho más dictada por las diferencias que existen en las propiedades físicas de los productos y en la disponibilidad de mano de obra calificada que por las simples diferencias en los costos de la mano de obra. La paradoja relacionada con el coeficiente de trabajo intensivo puede explicarse a satisfacción estableciendo que la mano de obra calificada es una forma de capital (humano), cuya escasez relativa en el mundo desarrollado es mucho menor que en el mundo en desarrollo, de manera similar a otras formas de capital. Por lo tanto, el comercio está aún dictado por las relaciones básicas de escasez relativa de los insumos primarios esenciales, si la mano de obra especializada se define también como una forma de capital.

La paradoja del coeficiente de mano de obra a la que nos hemos referido aquí difiere de la llamada paradoja de Leontief. Esta última es una cuasiparadoja, puesto que las exportaciones de los Estados Unidos con alta densidad de mano de obra, estaban basadas en la mano de obra y en los valores mixtos de ingresos inherentes a un modelo de insumo-producto y comparados con importaciones derivadas de manera similar. La paradoja de Leontief podría resolverse estableciendo que debido a la productividad de la mano de obra en los Estados Unidos, la remuneración al trabajo es varias veces -en promedio digamos unas tres veces- más alta que en los paises de donde provienen las importaciones; subsecuentemente, dicha remuneración fue corregida por este factor de tres, dando como resultado el descubrimiento de que las exportaciones de los Estados Unidos tenían un coeficiente de mano de obra mucho menor en términos de años-hombre que las importaciones.

La paradoja de la densidad de mano de obra que trato de explicar es verdadera. En el campo de los productos intensivos en trabajo, las importaciones en el mundo desarrollado tienen un coeficiente de mano de obra menor que su producción del mismo grupo de artículos finales fabricados en el mundo desarrollado, y que también exporta. Repitiendo de acuerdo con nuestras pruebas, la división internacional del trabajo se basa en la diferencia, entre los parses desarrollados $\mathrm{y}$ en desarrollo, de tres factores estrechamente interrelacionados:

1) las características físicas del producto (combinación de productos);

2) la habilidad complementaria que se requiere para esta producción (combinación de habilidades); $y$,

3) las técnicas de producción complementarias (combinación de tecnologías).

No obstante, la explicación proporcionada está todavía muy en línea con la teoría existente; los aspectos tecnológicos y políticos que se analizan brevemente a continuación establecen ciertos límites prácticos para este marco teórico. 
Considero que la explicación ofrecida aquí en relación con la paradoja del coeficiente de mano de obra es más satisfactoria que la explicación de Leontief basada en la remuneración al trabajo o en las diferencias de los costos; la nuestra se fundamenta en la escasez relativa de los factores básicos de la producción, particularmente de la mano de obra calificada, entre los países.

La conclusión más importante de este análisis no está en el hecho de que la explicación de la paradoja de la densidad de la mano de obra difiera, sino en que nuestro descubrimiento sea exactamente lo opuesto a la conclusión de Leontief; esto es, para ciertos bienes la especialidad de la producción del mundo desarrollado parece ser la de productos con mayor densidad de mano de obra, los cuales son también los de más alto coeficiente de habilidad manual, mientras que en los países en desarrollo, la especialización debe linearse en los de menor intensidad de mano de obra y menor coeficiente de habilidad manual. Este descubrimiento fue posible por el nivel de agregación más bajo utilizado en las investigaciones. Por lo tanto, es necesario diferenciar aún más los factores de la producción a fin de tener en cuenta la mano de obra calificada o adiestrada como factor diferente, o sea capital humano. Hacerlo está realmente más de acuerdo con un enfoque analítico microorientado que macroorientado, lo que señala claramente la necesidad de contar también con ambos enfoques.

\section{Aspectos tecnológicos.}

En la división internacional del trabajo la tecnologfa y el progreso tecnológico desempeñan un importante papel, no sólo como mercancías (servicios) para el comercio internacional, sino también como elementos que influyen y determinan una ventaja comparativa. El cambio tecnológico todavía es casi exclusivo de los países altamente desarrollados. Es tanto un fenómeno como una resultante de cierto escenario o ambiente económico, cultural y sociopolítico, y por lo tanto, se encuentra totalmente condicionado por este escenario de tal manera que, en nuestra opinión, se justifica hablar de un cambio, geotecnológico que resulta en una geotecnología, muy adecuada para la parte del mundo en la que se origina, pero muy poco adecuada para las naciones en desarrollo.

El cambio geotecnológico del mundo desarrollado confirma o restituye su ventaja comparativa en la producción de mercancías de alto contenido de mano de obra y ciertamente en las de elevada tecnología. Tiende a prolongar, e incluso a perpetuar la dependencia tecnológica del Tercer Mundo que implica dependencia económica y política. Es, quiérase o no, un vehículo de dominación y de implícito poder imperialista. Es, de manera particular, contra esta dependencia tecnológica contra lo que el Tercer Mundo más se rebela, y en realidad creo que la desigualdad tecnológica internacional, que conduce a una dependencia fundamental, es en gran medida básica y determinante para las relaciones entre las naciones del mundo desarrollado y las del mundo en desarrollo.

En un libro recientemente publicado, doy cuenta de los datos que descubrí en relación con las opciones tecnológicas y la división internacional del trabajo en la manufactura de calzado.* Sobre la base de microanálisis detallados de la

\footnotetext{
* Véase la referencia 4.
} 
operación y del nivel de operación de grupo empleado en el hormar de zapatos, encontré que cierto nivel de tecnología intermedia tendía a convertirse en subóptimo para cualquier relación salario-renta utilizada. En otras palabras, la altemativa de aplicar un mayor coeficiente de tecnología propendia a ser, y en la mayoría de los casos era, superior en eficiencia, independientemente de los precios de los factores de la producción. Por lo tanto, se eliminaba una tecnología básicamente adecuada para la mayor parte de las empresas de tamaño mediano del Tercer Mundo. Se presentó la siguiente secuencia de acontecimientos: los fabricantes de calzado de los países con altos costos de mano de obra que experimentaron una fuerte competencia de los países cuyos costos de mano de obra eran más bajos, se vieron obligados, para sobrevivir, a elevar la eficiencia de su producción. Por tanto, presionaron directa e indirectamente a los productores de tecnología para obtener maquinaria de mayor rendimiento. Estos últimos, por iniciativa propia, exploraban las necesidades de su clientela, lo que es una importante directriz en su esfuerzo tecnológico innovador. Pues to que la producción de maquinaria está sujeta a economías de escala, resulta muy provechoso aumentar el tamaño de los lotes de producción. La nueva maquinaria, al ahorrar mano de obra, puede también ahorrar capital si minimiza los costos mediante escalas óptimas de producción. La nueva maquinaria que ahorra mano de obra y capital por unidad de producción resulta atractiva por igual a los países avanzados y a los que se encuentran en desarrollo. Esto contrasta con una maquinaria que únicamente ahorra mano de obra y que en principio sólo interesaría a los países prósperos. ¿Qué está sucediendo fundamentalmente? Como respuesta a las ventajas comparativas en la producción de los países con costos más bajos de mano de obra, y las exportaciones que hace el Tercer Mundo de estos productos, el progreso tecnológico recibe un estímulo en el mundo desarrollado para ofrecer técnicas de producción que respondan al reto. Como resultado se obtienen técnicas de producción que permiten ahorrar mano de obra y que pueden resultar también en ahorro de capital. Este último efecto puede lograrse también fijando el precio de la nueva maquinaria de tal manera que signifique un ahorro de capital y estimule la demanda mundial tomando en cuenta el grado de obsolescencia económica, lo que incide para elevar la escala de producción que justifica retrospectivamente su precio inicial de mercado. El incremento de la demanda del Tercer Mundo por esta maquinaria superior podría ser el elemento que hiciera bajar el coeficiente de capital por unidad de producción hasta un nivel crítico, o sea, inferior al de la maquinaria anterior. El Tercer Mundo proporciona involuntariamente el incremento crítico de la demanda, lo que hace que la nueva maquinaria sea económicamente superior y que ofrezca ganancias tanto al productor de la tecnología cuanto a los fabricantes de calzado en los países en los que los costos de mano de obra son elevados. Así, los fabricantes de calzado del mundo en desarrollo proporcionan esencialmente una subvención a los que manufacturan calzado en los países desarrollados, restituyendo y mejorando la ventaja comparativa de estos últimos. Sin embargo, aparece una tecnología menos adecuada para el Tercer Mundo, y que deteriora la ventaja comparativa de éste en dicha producción. Este mecanismo tiene una lógica que raya en el cinismo. Proporciona una justificación completa, en nuestra opinión, para que el Tercer Mundo al pro- 
mover sus exportaciones adopte medidas que rectitiquen este subsidio involuntario e injustificado que da a sus competidores del mundo desarrollado.*

El mecanismo interdependiente que hemos descrito, basado parcialmente en pruebas empíricas y en un marco de referencia hipotético, explicativo y complementario, ilustra la fuerza desestabilizadora del progreso geotecnológico, que da como resultado una persistente dependencia tecnológica del Tercer Mundo, con los nexos económicos y políticos que implica.

Esta influencia del cambio tecnológico en los costos comparativos de producción, por su misma naturaleza que permite ahorrar a la rez mano de obra y capital, parece estar en contradicción con el fenómeno de la paradoja de la densidad de mano de obra ya analizada y explicada. Para la producción de mercancías uniformes y poco complejas que no requieren de mucha habilidad operativa, el Tercer Mundo parece tener una ventaja comparativa en su fabricación utilizando maquinaria moderna. Si es así, la nueva tecnología para la producción en grandes cantidades que permite economizar mano de obra y capital, puede ser más útil, o por lo menos tan útil, para el mundo en desarrollo como para el mundo desarrollado. Una tecnología que permite ahorrar capital representa más ventajas en regiones en las que el capital es más escaso que en las que no lo es. Y no me parece que haya aquí contradicción alguna. Lo que debe recordarse es que dos enunciados, por contradictorios que parezcan, pueden resultar verdaderos en la compleja realidad que los economistas a menudo simplifican demasiado.

Aun cuando rebase los límites previstos, puede ser útil aclarar un poco más lo que tengo en mente, ya que ni en el documento que aquí se resume, ni en el libro, ha sido suficientemente desarrollado.

Empecemos por el otro extremo. De acuerdo con la paradoja de la densidad de mano de obra, los países que tienen altos costos de mano de obra disponen de una ventaja comparativa en la producción físicamente más compleja o en la especializada de manera más general, lo que implica no sólo un coeficiente relativamente alto en la calificación del personal, sino también en la fabricación de bienes de trabajo intensivo. Sin embargo, las especializaciones en la producción nunca son fijas ni se dan a un tiempo. Gradualmente se construye también en el mundo en desarrollo una base de mano de obra calificada, sobre todo en aquellos países que se iniciaron temprano en la producción industrial de mercancias con densidad relativamente elevada de mano de obra. Por consiguiente, no sólo es imaginable, sino que de hecho ya ocurre que esta ventaja comparativa del mundo desarrollado se esté erosionando. El mecanismo especial del cambio tecnológico descrito anteriormente no sólo es aplicable a los productos con características físicas relativamente simples, sino también a los más complejos y poco comunes. Un buen ejemplo es el caso de la acelerada introducción de la microelectrónica a la tecnología convencional para la producción de artículos con coeficiente relativamente alto de mano de obra, como son las prendas de vestir y el calzado. Esta nueva tecnología es particularmente conveniente para la fabricación de los productos especializados del mundo industrializado, la cual permite economizar mano de obra simple y calificada, y también será ahorradora de ćapital.

* Véase la referencia 3. 
Sin embargo, se trata de una geotecnología muy propia del mun do avanzado y resultaría muy inoportuno que se difundiera rápidamente al Tercer Mundo, poniéndolo más en posibilidades de economizar capital, de acuerdo con el mecanismo descrito anteriormente. Si así fuera, funcionaría sólo para beneficio del mundo desarrollado y en contra del Tercer Mundo. A pesar de que los fabricantes de calzado del mundo marginado proporcionaran el aumento decisivo en la demanda de la nueva tecnología, los fabricantes de calzado del mundo privilegiado serian los que obtendrían los beneficios. Esto se explica por las siguientes razones:

- La nueva tecnología es una geotecnología de las naciones desarrolladas, mucho más adecuada para los usuarios de esta región que para el Tercer Mundo.

- A pesar de que la nueva tecnología reduce la necesidad de la mano de obra calificada, en última instancia crea una dependencia de otras especialidades aún más escasas:

a) programadores para computación;

b) planificadores de producción interna;

c) diseñadores de modas; $y$,

d) especialistas en mercados.

A pesar de que la nueva maquinaria permite un ahorro de capital, esto depende en gran medida de:

a) su precio de adquisición comparado con el de la maquinaria convencional,

b) su grado de utilización efectiva, que depende de numerosas condiciones interrelacionadas, entre las que se cuentan las mencionadas anteriormente.

Por estas razones, es poco probable que la nueva tecnología pueda tener efecto, por algún tiempo, en la actual división internacional del trabajo, incluso si llegará a difundirse en el Tercer Mundo. De hecho, esta difusión tan sólo reforzaría la actual producción especializada del mundo desarrollado, ya que recibe una nueva tecnología que es relativamente más adecuada para ella que para los países en desarrollo e inclusive más barata. Por lo tanto, la maquinaria controlada electrónicamente, utilizada para manufacturar calzado, ropa y algunos otros artículos con coeficiente de mano de obra relativamente alto (como ciertos productos metálicos) confirmará por algún tiempo la actual división internacional del trabajo en cuanto a estas mercancías.

Para mayor claridad, lo explicaré más ampliamente. Significa que esta nueva tecnología de control electrónico tendrá dos importantes impactos directos en la división internacional del trabajo en relación con los productos que se analizan:

1. Detiene, por el momento, el proceso lógico de la división internacional del trabajo, el cual habría continuado de no aparecer este cambio tecnológico microelectrónico.

2. La división internacional del trabajo alcanzada hasta ahora no dará marcha atrás, ya que la nueva tecnología favorece menos, por ahora, a los productos comunes más simples producidos en grandes series. 
En otras palabras, la nueva tecnología contribuye a confirmar y a consolidar la actual división internacional del trabajo; beneficia más a las regiones de alta tecnología y de costos elevados de mano de obra que a las regiones con costos de mano de obra relativamente menores y de baja tecnología. Esto confirma nuestra posición de que el cambio tecnológico está geocondicionado y funciona para beneficio de las zonas en las que se origina y, por lo tanto, puede ir en contra de los intereses económicos, de comercio exterior, tecnológicos y políticos del Tercer Mundo.

Sin embargo, también creo que la nueva tecnología puede generar nuevas oportunidades para una división internacional del trabajo en relación con productos que hasta ahora no se encuentren afectados por este tipo de especialización del comercio y la producción. Un ejemplo es la producción de tecnología convencional incorporada caracterizada por requerimientos mecánicos de alta precisión. Sin duda la nueva tecnología ha encontrado y en el futuro seguirá encontrando cada vez mayores aplicaciones selectivas en países de reciente industrialización. Una de dichas aplicaciones es la producción de tecnologias convencionales, las cuales en realidad son especialidades producidas en pequeños lotes. Estas nuevas tecnologías contribuyen a facilitar las especificaciones de precisión que a menudo constituían un obstáculo para los países en desarrollo. Sería muy importante que esta hipótesis se confirmara, ya que esto implicaría que un cambio geotecnológico en el mundo desarrollado cambiaría la división internacional del trabajo a través de producción de tecnologías incorporadas convencionales, lo que reduciría la dependencia tecnológica de los países en desarrollo.

\section{Aspectos politicos}

El proceso para alcanzar una independencia real, que implica también un grado aceptable de dependencia tecnológica, económica y política, es demasiado lento para satisfacer a los países en desarrollo. En los años setenta, el malestar producido por la lentitud de este proceso, junto con sus correspondientes frustraciones, ocasionó una mayor inestabilidad en las relaciones Norte-Sur y una tendencia a emplear otras políticas y estrategias para transferir riqueza de la parte opulenta a la más pobre del mundo. En realidad, el comportamiento del Tercer Mundo a nivel internacional se caracteriza más ahora por el empleo de algunos medios poco convencionales y legalmente dudosos para atraer la transferencia de riqueza, que los utilizados anteriormente. En la actualidad, ciertos gobiernos practican el chantaje, el terrorismo, la extorsión y otros medios similares. En esencia, el deseo de alcanzar un nuevo orden internacional es un intento por llegar a una transferencia de la riqueza ordenada y negociada internacionalmente, con el fin de evitar prácticas desorganizadas e ilegales que aménazan la estabilidad política mundial entre el Norte y el Sur.* No obstante, inclusive los progresos realizados en las negociaciones por el nuevo orden internacional están determinados esencialmente por los cambios ocurridos en las relaciones fundamentales de estruc-

* Véanse las referencias 6 y 7 . 
turas del poder a nivel internacional y por el interés propio de ambos grupos de países. De hecho, un nuevo orden internacional podrá funcionar sólo si estuviera basado en una verdadera relación estructural y fundamental de poder, para que pueda ser eficaz. El comercio mundial y la división internacional del trabajo son superestructuras basadas en estas relaciones de poder más fundamentales, subyacentes e internacionales. La interdependencia entre las naciones, en un sentido económico y tecnológico, puede existir sólo si está basada en un equilibrio de poder político, pluralista e internacional. La llamada interdependencia del sistema internacional, como funcionó en el período 1850-1914 y en menor grado de 1919 a 1939, estuvo basada en la supremacía europea y por consiguiente era, de hecho, una relación de dependencia. El incremento del poder político de la India, China $\mathrm{y}$ algunos países árabes y latinoamericanos podría dar como resultado una estructura de poder internacional más pluralista que estimulara la interdependencia económica y tecnológica. Sin embargo, las relaciones de causa y efecto son, como de costumbre, complicadas, ya que ciertas formas de desarrollo económico y tecnológico hacen aumentar a su vez el poder político de los países.

\section{Referencias}

1. Boon, G.K. Factores Fisicos y Humanos en la Producción. North Holland Publishing Company, Amsterdam, 1964, Fondo de Cultura Económica, México, D.F., 1970.

2. Technology and Sector Choice in Economic Development, SythoffNoordhoff International Publishers, Alphen, 1978. (Próxima edición en español de El Colegio de México, 1983).

3. - Technology and Employment in Footwear Manufacturing, páginas 156-164, Sythoff-Noordhoff, 1980.

4. Technology Transfer in Fibres. Textile and Apparel, vea tema index geo-technologia,-- técnicas. Sythoff-Noordhoff, 1981. (Próxima edición en español de El Colegio de México, 1983).

5. - Technology Behaviour and Assessment, pendiente publicación.

6. Brandt, W. et al. North-South. A Program for Survival, Pan Books, London, Sydney, 1980. (Informe Brandt; hay edición en español).

7. Tinbergen, J. et al. Reestructuración del orden internacional (Informe RIO). México, Fondo de Cultura Económica, 1976. 